\title{
Downregulated long non-coding RNA TCONS_00068220 upregulates apoptosis in gastric cancer cells
}

\author{
ZHIWEI ZHAO ${ }^{1}$, JUNLAI XUE ${ }^{2}$, YAN SONG $^{1}$, TIANYOU LIU $^{3}$ and DAXUN PIAO ${ }^{3}$ \\ Departments of ${ }^{1}$ General Surgery and ${ }^{2}$ Traditional Chinese Medicine, China-Japan Friendship \\ Hospital of Jilin University, Changchun, Jilin 130033; ${ }^{3}$ Department of General Surgery, First Affiliated \\ Hospital of Harbin Medical University, Harbin, Heilongjiang 150001, P.R. China
}

Received May 9, 2016; Accepted August 8, 2017

DOI: $10.3892 / 01.2017 .6977$

\begin{abstract}
Long non-coding RNAs (lncRNAs) are emerging as a fundamental class of biological effect or molecules that perform pivotal functions in the regulation of the genome. With advances in bioinformatics and genomics, extensive identification and characterization of lncRNAs is now possible. They regulate cellular growth, differentiation and apoptosis. Dysregulation of IncRNAs has been associated with numerous types of human cancer. In the present study, the expression profile of differentially expressed genes (DEGs) and lncRNAs in gastric cancer (GC) samples and normal tissue samples was evaluated with bioinformatics. The biological functions of the predicted IncRNA TCONS_00068220 were focused on; the DEGs co-expressed with TCONS_00068220 were enriched in cancer-associated pathways. TCONS_00068220 was demonstrated to be upregulated in GC tissues and cell lines compared with normal controls. In addition, an increased rate of apoptosis was observed in NCI-N87 cells following transfection with small interfering RNA against TCONS_00068220. These data suggest that TCONS_00068220 may be associated with the pathogenesis of GC, and it may serve as a potential therapeutic target.
\end{abstract}

\section{Introduction}

Gastric cancer (GC) is ranked as the fourth most commonly diagnosed and the second most lethal type of malignancy worldwide (1). A total of 989,600 new cases of GC and 738,000 mortalities are estimated to occurglobally per year, accounting for $8 \%$ of all cases of cancer and $10 \%$ of cancer mortality (2). In the majority of patients, GC is diagnosed at an advanced stage at which malignant proliferation, extensive

Correspondence to: Dr Yan Song, Department of General Surgery, China-Japan Friendship Hospital of Jilin University, 126 Xiantai Avenue, Changchun, Jilin 130033, P.R. China

E-mail: yansongysy@163.com

Key words: gastric cancer, long non-coding RNAs, RNA-sequencing data, TCONS_00068220, apoptosis invasion, and lymphatic metastasis have already occurred (3). Despite the progress in chemotherapy, radiotherapy and surgical techniques for GC in recent decades, the outcomes forpatients with $\mathrm{GC}$ remain poor, and 5-year overall survival rates are $<25 \%(4,5)$. Therefore, an improved understanding of the pathogenesis and identification of the molecular alterations is essential for the development of diagnostic markers and therapeutic targets to allow the sensitive diagnosis and effective therapy of GC $(3,6)$.

Long non-coding RNAs (IncRNAs), measuring >200 nucleotides in length, are a class of non-coding RNA molecule with limited or no protein-coding capacity (7). They are associated with a variety of biological processes, including cell proliferation, cell cycle, differentiation and apoptosis, primarily by the regulation of gene expression through chromatin remodeling, RNA maturation, transport and protein synthesis (8). Advances in the depth and quality of transcript me sequencing have revealed a range of IncRNAs classes dysregulated in various human diseases, particularly cancer (9-11). H19 is a member of a highly-conserved cluster of imprinted genes, and was the first lncRNA to be associated with cancer (12). Previous studies have indicated that $\mathrm{H} 19$ exhibits dysregulated expression in GC $(13,14)$, hepatocellular carcinoma (15), bladder cancer (16), breast cancer (17) and esophageal cancer (18), and that itmay cause tumor promoting and suppressing effects (19). Niu et al (20) screened IncRNA expression profilesfrom colorectal cancer and adenoma samples, and identified a novel lncRNA, AK027294, which was associated with cell proliferation, migration and apoptosis in colorectal cancer cells. Li et al (21) and Song et al (22) investigated the lncRNA expression patterns in GC with a microarray, and identified 135 differentially expressed lncRNAs in GC tissues compared with non-tumor adjacent tissues (NATs). Furthermore, the expression level of gastric cancer-associated transcript 1 (GACAT1) was associated with the lymph node metastasis, distant metastasis, tumor node metastasis stage and differentiation of GC (23). By analyzing RNA-sequencing (seq) and public microarray data, Park et al (24) identified 31 differently expressed transcripts in GC, including BM742401, which was verified as a potential lncRNA marker and therapeutic target in subsequent experiments.

In the present study, the differentially expressed genes (DEGs) and lncRNAs in GC samples compared with normal tissue samples were screened using bioinformatics analysis. 
lncRNA-DEG pairs were identified, and pathway enrichment analysis was performed for the DEGs co-expressed with each lncRNA. Based on our previous data (25), the present study focused on the IncRNA TCONS_00068220, which was suspected to be associated with GC pathogenesis. TCONS_00068220 encodes a 1,281-nt RNA that contains 3 exons at the human genome locus 8q11.21. Further functional studies of TCONS_00068220 in the present study indicated that the down regulation of TCONS_00068220 may promote apoptosis in GC cells.

\section{Materials and methods}

Bioinformatics analysis. The GSE41476 RNA-seq data was obtained from the Gene Expression Omnibus (GEO; http://www.ncbi.nlm.nih.gov/geo/). The expression profilesof 3 primary cell culture samples from GC tissue and 2 normal tissue samples from GSE41476 were included in the dataset. The RNA-seq data were compared with human genome 19 from the University of California Santa Cruz (UCSC) database (http://genome.ucsc.edu) and aligned to achieve transcript tome assembly. Subsequently, comparative genomics methods were utilized to predict the novel lncRNAs. DEGs and differentially expressed known and predicted lncRNAs were screened usingan adjusted P-value of $<0.05$ and $\mid \log$ fold-change $(\mathrm{FC}) \mid>1$ as the cut-off criteria. The association between DEGs and differentially expressed lncRNAs was calculated; a Pearson's correlation coefficient $>0.99$ was used as the cut-off criterion for DEG-IncRNA pairs. Subsequently, Kyoto Encyclopedia of Genes and Genomes (KEGG; www.genome.jp/kegg/) pathway enrichment analysis was conducted for the DEGs associated with each IncRNA. The detailed procedures for bioinformatics analysis have been described in a previous study (25).

Tumor tissues and cell lines. GC tissues and matched NATs were collected from 15 patients who had undergone the surgical resection of GC at the First Affiliated Hospital of Harbin Medical University (Harbin, China) and the People's Hospital of Heilongjiang Province (Harbin, China) between January 2015 and March 2015. The mean age of the patients was 59.5 years (range $47-74$ years) and the ratio of males to females was 9:6. No local or systemic treatment had been administered to the patients prior to surgery. The NAT samples were obtained $5 \mathrm{~cm}$ away from the tumor margin. All tissue samples were snap-frozen in liquid nitrogen immediately following resection and stored at $-80^{\circ} \mathrm{C}$ until RNA extraction. The study was approved by the Ethics Committee of First Affiliated Hospital of Harbin Medical University and was performed in compliance with the Declaration of Helsinki. Written, informed consent was obtained from the patients involved in the study.

Human GC cell lines (including MKN-45, SNU-484 and NCI-N87) and the human immortalized gastric epithelial cell line GES-1 were purchased from the Biochemistry and Cell Biology at the Chinese Academy of Sciences (Shanghai, China). All cell lines were cultured in Dulbecco's modified Eagle's medium (Gibco; Thermo Fisher Scientific, Inc., Waltham, MA, USA) supplemented with $10 \%$ fetal bovine serum (FBS) (Gibco; Thermo Fisher Scientific, Inc.), 100 U/ml penicillin, and $100 \mathrm{mg} / \mathrm{ml}$ streptomycin (Gibco; Thermo Fisher
Scientific, Inc.). The cells were incubated at $37^{\circ} \mathrm{C}$ in a humidified atmosphere with $5 \% \mathrm{CO}_{2}$.

RNA extraction and reverse transcription-quantitative polymerase chain reaction $(R T-q P C R)$ analyses. According to the manufacturers' protocols, total RNA was extracted from tissues and cultured cells with RNAiso Plus (Takara Biotechnology Co., Ltd., Dalian, China), and reverse transcription was performed using PrimeScript ${ }^{\mathrm{TM}}$ RT Master Mix (Takara Biotechnology Co., Ltd.). qPCR was performed using PowerSYBR Green PCRMastermix (AppliedBiosystems, Thermo Fisher Scientific, Inc.) according to the manufacturer's protocol. The PCR primers for TCONS_00068220 and GAPDH (Synbio Technologies LLC, Suzhou, China) were as follows: TCONS_00068220 forwards, 5'-AATACCAGAGGC TCCAAGAACAG-3', and reverse, 5'-CGACTACATAAG GGCTTAGAAACG-3'; GAPDH forwards, 5'-TGACAACTT TGGTATCGTGGAAGG-3', and reverse, 5'-AGGCAGGGA TGATGTTCTGGAGAG-3'. qPCR was performed in a total reaction volume of $20 \mu \mathrm{l}$, including $2 \mu \mathrm{l}$ RT products, $0.4 \mu \mathrm{l}$ of forward primers, $0.4 \mu \mathrm{l}$ of reverse primers, $10 \mu \mathrm{l}$ of $2 \mathrm{X}$ SYBR Green Mix and 7.2 $\mu \mathrm{l}$ RNase-free $\mathrm{dH}_{2} \mathrm{O}$. The reactions were performed with the following protocol: An initial denaturation of $10 \mathrm{~min}$, followed by 40 cycles of $95^{\circ} \mathrm{C}$ for $15 \mathrm{sec}$ and $60^{\circ} \mathrm{C}$ for $60 \mathrm{sec}$. Relative gene expression was calculated using the comparative cycle threshold method (value of $2^{-\Delta \Delta \mathrm{Cq}}$ ) (26) with GAPDH as an endogenous control. The RT-qPCR assays and data collection were performed using an ABI ViiA7 instrument (Applied Biosystems; Thermo Fisher Scientific, Inc.). Each sample was analyzed in triplicate.

Downregulation of TCONS_00068220. Small interfering RNA (siRNA) for TCONS_00068220 (si-TCONS_00068220, 5'-CCUGUAAUAAACGUCACAATT-3') and negative control (si-NC, 5'-UUCUCCGAACGUGUCACGUTT-3') were synthesized by Shanghai GenePharma Co., Ltd. (Shanghai, China). NCI-N87 cells were transfected with si-TCONS_00068220 or si-NC using Lipofectamine $2000{ }^{\circledR}$ transfection reagent (Invitrogen; Thermo Fisher Scientific, Inc.) according to the manufacturer's protocol.

Cell viability assay. NCI-N87 cells transfected with si-NC or si-TCONS_00068220 $\left(1.5 \times 10^{5}\right.$ cells/well $)$ were seeded in 12 -well plates. Trypan blue exclusion assays were performed to assess cell viability at 1,2,3, 4 and 5 days. A mixture of adherent and suspended cells was collected, mixed with $0.4 \%$ trypan blue solution (Beyotime Institute of Biotechnology, Haimen, China), added into a hemocytometer and observed under a light microscope (magnification, x 100; BX43; Olympus Corporation, Tokyo, Japan). Blue-stained cells were counted as dead, and cell viability (\%) was expressed as [1-(dead cell number/the total cell number)] $\mathrm{x} 100 \%$. The experiments were performed in triplicate.

Apoptosis analysis by flow cytometry. NCI-N87 cells $\left(1 \times 10^{5}\right)$ were harvested with Trypsin and washed with ice-cold PBS 3 days subsequent totransfection. Apoptotic cell death was determined using a fluorescein isothiocyanate-Annexin $\mathrm{V}$ Apoptosis Detection kit I (BD Pharmingen; BD Biosciences, Franklin Lakes, NY, USA) according to the manufacturer's 
protocol. Samples were stained for $30 \mathrm{~min}$ at room temperature in the dark. The stained specimens were then analyzed by FACSC alibur flow cytometry with Cell Quest software version 3.0 (BD Biosciences). Experiments were performed in triplicate.

Nuclear staining with DAPI. NCI-N87 cells (1x105 cells/well) were seeded in a 12 -well plate with a glass slide at the bottom of each well, and then cultured and transfected with siRNAs as previously described. Following 3 days of incubation, the cells were washed in PBS and fixed with $1 \mathrm{ml} 4 \%$ paraformaldehyde solution at room temperature for $20 \mathrm{~min}$. The fixed cells were subsequently washed with PBS and stained with VECTASHIELD ${ }^{\circledR}$ Antifade Mounting Medium with $1.5 \mu \mathrm{g} / \mathrm{ml}$ DAPI (Vector Laboratories, Inc., Burlingame, CA, USA) at room temperature in the dark for $5 \mathrm{~min}$. Nuclear morphology of cells was examined by a confocal laser scanning platform (TCS SP8; Leica Microsystems GmbH, Wetzlar, Germany).

Western blot analysis. NCI-N87 cells were seeded at a density of $3 \times 10^{5}$ cells per well in a 6 -well plate and transfected with si-NC or si-TCONS_00068220. The cells were collected at 3 days after transfection and resuspended in RIPA Lysis Buffer III (Sangon Biotech Co., Ltd., Shanghai, China). The concentration of total protein was quantified by bicinchoninic acid (BCA) method. The protein extracts $(20 \mu \mathrm{g} /$ well) were resolved by $10 \%$ SDS-PAGE and transferred to a polyvinylidene difluoride membrane, followed by blocking with PBS and Tween-20 solution supplemented with 5\% non-fat milk at room temperature for $1.5 \mathrm{~h}$. The membrane was incubated with primary antibodies against p17-specific cleaved caspase-3 (cat. no., 25546-1-AP; dilution, 1:500) and GAPDH (cat. no., 60004-1-Ig; dilution, 1:2,000; both ProteinTech Group, Inc., Chicago, IL, USA) at $4^{\circ} \mathrm{C}$ overnight, and then incubated with horseradish peroxidase-conjugated goat anti-rabbit IgGsecondary antibodies (cat. no., 111-035-045; dilution, 1:5,000; Jackson ImmunoResearch Laboratories, Inc., West Grove, PA, USA) at room temperature for $1.5 \mathrm{~h}$. The ChemiDoc ${ }^{\mathrm{TM}}$ XRS+ system (Bio-Rad Laboratories, Inc., Hercules, CA, USA) was used to visualize the bands.

Statistical analysis. Data were expressed as the mean \pm standard deviation (SD) from three separate experiments. The western blot analysis results are presented from a representative experiment. Statistical significance was determined using the two-tailed Student's t-test. Multiple groups were analyzed using a one-way analysis of variance followed by a Newman-Keuls test. All statistical analyses were performed using SPSS 17.0 software (SPSS Inc., Chicago, IL, USA). $\mathrm{P}<0.05$ was considered to indicate a statically significant difference.

\section{Results}

TCONS_00068220 is differentially expressed in GC compared with NATs, and co-expressed DEGs of TCONS_00068220 are enriched in cancer-associated pathways. Compared with NATs, a total of 2,625 differentially expressed transcripts' including 34 known lncRNAs, 5 predicted lncRNAs and 2,586 mRNAs) were identified in the GC samples (Fig. 1A; Table I).
Table I. Differentially expressed long non-coding RNAs in gastric cancer tissue compared with normal tissue samples.

\begin{tabular}{llll}
\hline Name & \multicolumn{1}{c}{$\mid \log \mathrm{FCl}$} & P-value & Q-value \\
\hline TCONS_00028798 & $1.79769 \mathrm{E}+308$ & $2.74 \times 10^{-4}$ & $5.26 \times 10^{-3}$ \\
TCONS_00038095 & 2.79182 & $5.24 \times 10^{-3}$ & $4.69 \times 10^{-2}$ \\
TCONS_00058340 & $1.79769 \mathrm{E}+308$ & $2.98 \times 10^{-3}$ & $3.13 \times 10^{-2}$ \\
TCONS_00068220 & 4.92069 & $1.57 \times 10^{-3}$ & $1.94 \times 10^{-2}$ \\
TCONS_00037998 & 4.74522 & $4.84 \times 10^{-4}$ & $8.05 \times 10^{-3}$ \\
\hline
\end{tabular}

FC, fold-change.

Following the identification of lncRNA-DEG pairs, KEGG pathway enrichment analysis was conducted for the DEGs co-expressed with each lncRNA (Fig. 1B). Based on this analysis, it was revealed that the DEGs co-expressed with the predicted lncRNA TCONS_00068220 were enriched in cancer-associated processes, including 'bladder cancer', 'cell adhesion molecules' (CAMs), 'chemokine signaling pathway' and 'natural killer cell-mediated cytotoxicity' (Table II) suggesting that TCONS_00068220 may serve a crucial biological function in GC cells.

TCONS_00068220 expression is upregulated in human GC tissues and cell lines. To assess the role of TCONS_00068220 in GC genesis and progression, the TCONS_00068220 expression level was examined in GC tissues and cell lines with RT-qPCR. The TCONS_00068220 levels were upregulated in $86.7 \%$ of GC tissues compared with NATs (Fig. 2A). The median fold change was 3.16 . The mean $\pm \mathrm{SD} \Delta^{\mathrm{Cq}}$ value for TCONS_00068220 in GC tissues was 9.70 1.97 , whereas it was $11.46 \pm 2.91$ for NATs (Fig. 2B).

It was then verified that the expression of TCONS_00068220 was increased in two GC cell lines (MKN-45 and NCI-N87) compared with the expression in the non-cancer GES-1 cells (Fig. 2C; $\mathrm{P}<0.05$ ). These data indicated that abnormal TCONS_00068220 expression may be associated with the genesis and progression of GC.

TCONS_00068220 downregulation inhibits the viability of $G C$ cells. RNA interference is an effective strategy for inhibiting gene expression in cultured cells (27). To verify whether TCONS_00068220 expression was associated with GC progression, siRNAs were used to downregulate TCONS_00068220 expression in the NCI-N87 cell line. The knockdown effect was confirmed by RT-qPCR analysis (Fig. 3A). Atrypan blue exclusion assay indicated that the rate of viable cells was reduced compared with cells transfected with the si-NC, and widespread cell death was observed at 3 days after transfection with si-TCONS_00068220 (Fig. 3B).

TCONS_00068220 downregulation promotes the apoptosis of GC cells. To confirm that TCONS_00068220 downregulation promoted GC cell apoptosis, flow cytometry analysis was performed. As demonstrated in Fig. 4A and B, when TCONS_00068220 expression was downregulated with siRNA, the rate of apoptosis was markedly increased. Based 
Table II. Top 10 enriched Kyoto Encyclopedia of Genes and Genomes pathways for the differentially expressed genes co-expressed with TCONS_00068220.

\begin{tabular}{lcll}
\hline Name & Genes, $\mathrm{n}$ & Associated genes & P-value \\
\hline Cell adhesion molecules & 15 & CD2, CD28 & $3.10 \times 10^{-6}$ \\
Chemokine signaling pathway & 15 & CCL5, CCR1 & $2.02 \times 10^{-4}$ \\
Bladder cancer & 5 & MMP9, MYC & $5.61 \times 10^{-3}$ \\
Natural killer cell mediated cytotoxicity & 8 & BID, ICAM2 & $3.38 \times 10^{-2}$ \\
Intestinal immune network for IgA production & 8 & CD28, ICOSLG & $4.17 \times 10^{-5}$ \\
Staphylococcus aureus infection & 8 & C1QB, C1QC & $1.14 \times 10^{-4}$ \\
Leishmaniasis & 9 & ITGB1, MAPK12 & $1.43 \times 10^{-4}$ \\
Type I diabetes mellitus & 7 & CD28, PRF1 & $1.49 \times 10^{-4}$ \\
Allograft rejection & 6 & CD28, HLA-DMB & $4.61 \times 10^{-4}$ \\
Viral myocarditis & 8 & BID, CD28 & $6.24 \times 10^{-4}$ \\
\hline
\end{tabular}

A

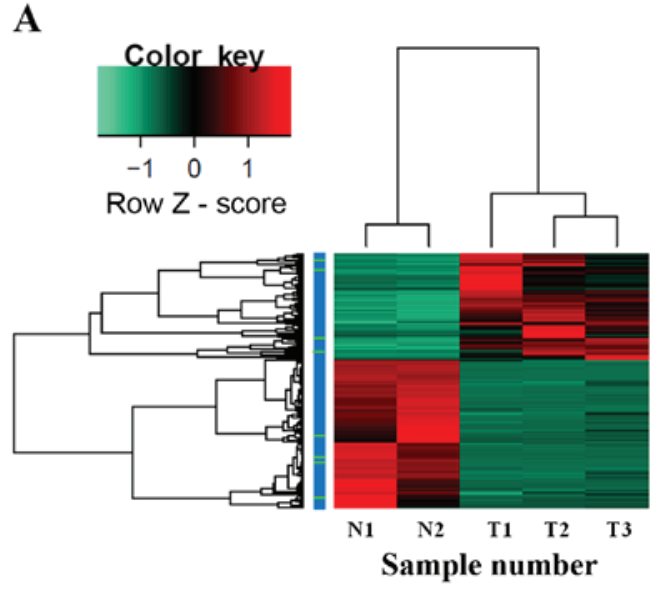

- DEGs

- Differentially expressed IncRNAs
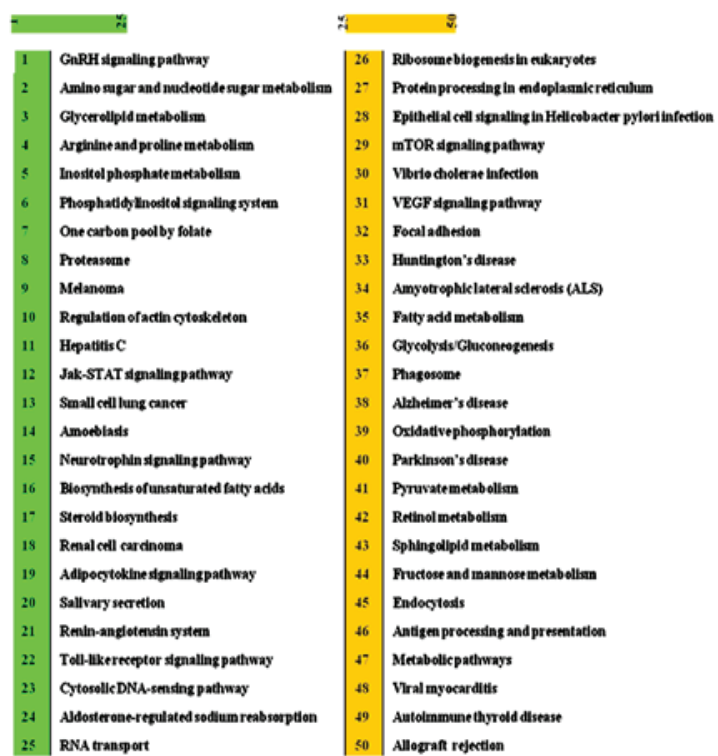

B

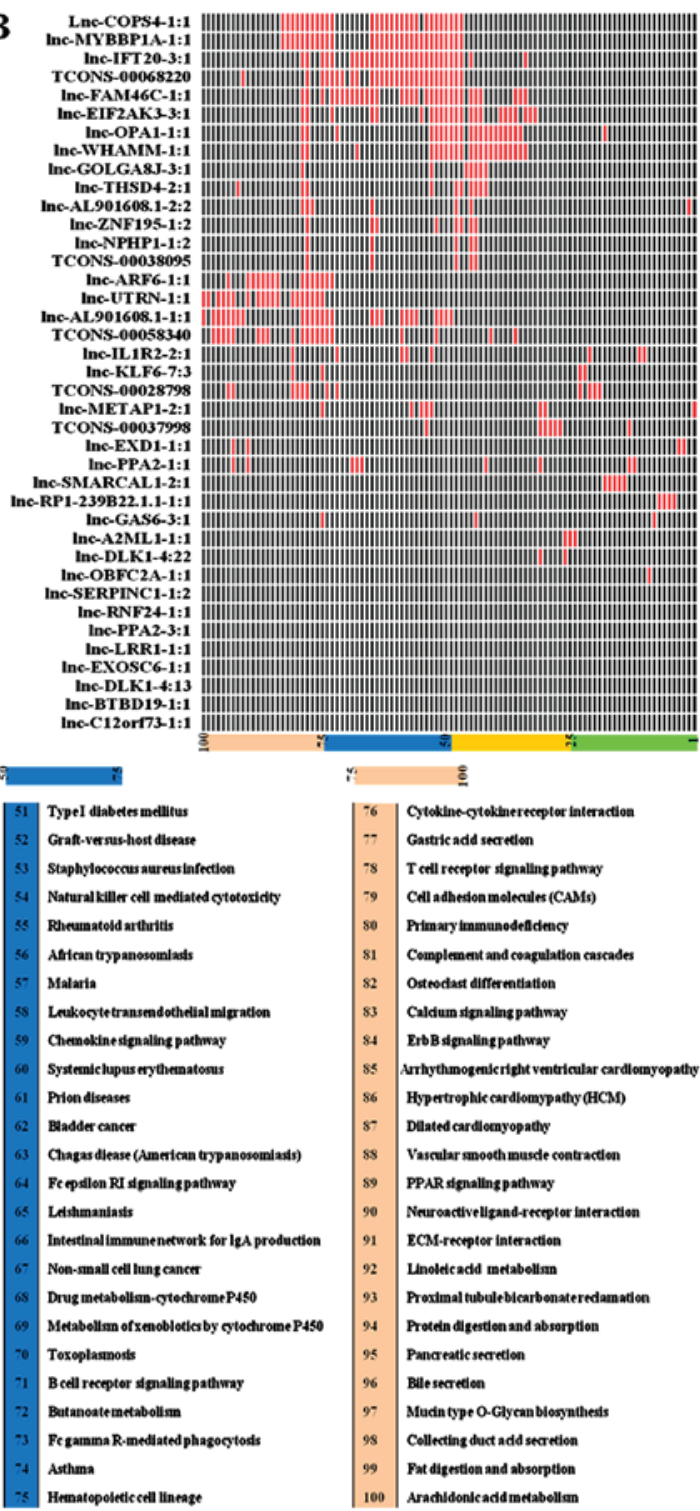

Figure 1. Expression profile screening and KEGG pathway enrichment map. (A) Clustering diagram of DEGs and differentially expressed lncRNAs between $3 \mathrm{GC}$ samples (T1, T2, T3) and 2 normal tissue samples (N1, N2). In the heatmap, red indicates high relative expression, and green indicates low relative expression. (B) KEGG enrichment map of the DEGs co-expressed with lncRNAs. The enriched KEGG pathways of the DEGs co-expressed with each lncRNA are indicatedby a red box. KEGG, Kyoto Encyclopedia of Genes and Genomes; DEGs, differentially expressed genes; lncRNAs, long non-coding RNAs; GC, gastric cancer. 
$\mathbf{A}$

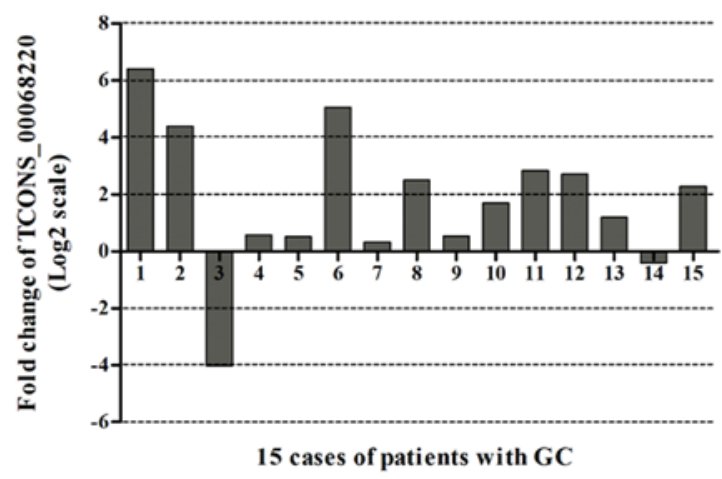

C
B

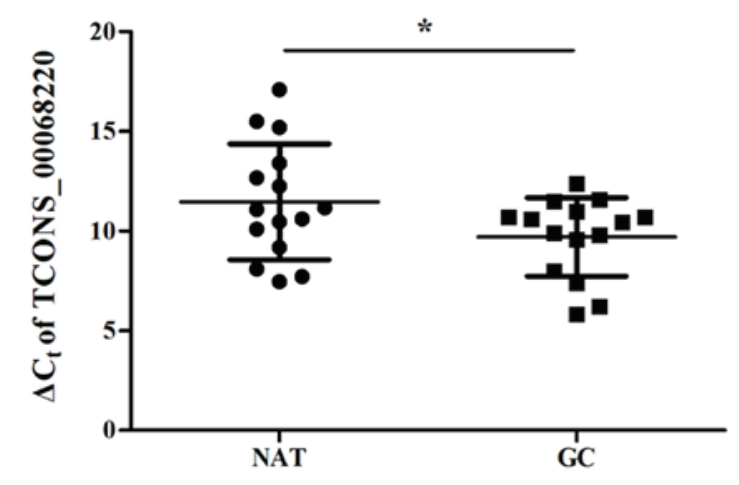

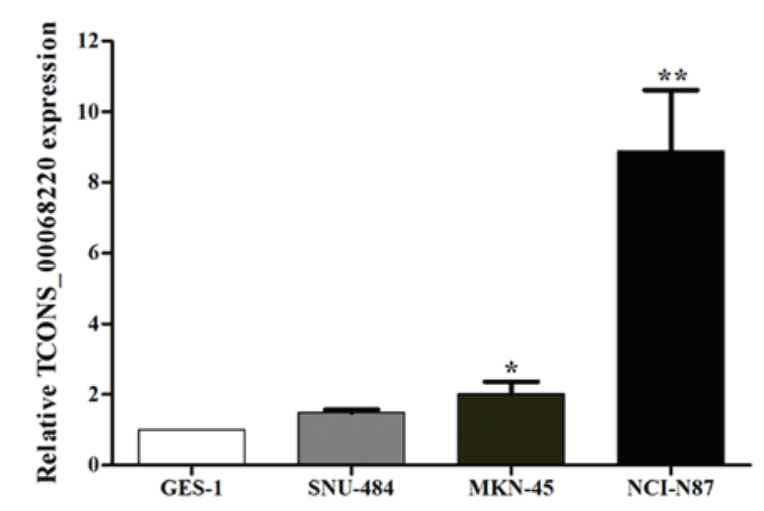

Figure 2. Expression of TCONS_00068220 in GC tissues and cell lines. (A) The relative expression of TCONS_00068220 in GC tissues compared with NATs. Data are presented as $\log$ base 2 of the fold change in GC tissue relative to the corresponding NAT. (B) $\Delta^{\mathrm{Ct}}$ values for TCONS_00068220 in GC tissues and NATs relative to GAPDH. (C) Expression levels of TCONS_00068220 in GC cell lines compared with the immortalized human gastric epithelial GES-1 cell line. Data are presented as mean \pm standard deviation. ${ }^{*} \mathrm{P}<0.05$ and ${ }^{* *} \mathrm{P}<0.01$ compared with NATs or GES-1. GC, gastric cancer; NAT, non-tumor adjacent tissue.

on observations of nuclear morphology, apoptotic cells were increased in si-TCONS_00068220 group compared with si-NC group (Fig. 4C). Additionally, the expression of caspase-3, a key mediator of apoptosis (28), was detected by western blot analysis. The results revealed that the expression of cleaved caspase-3 was enhanced in NCI-N87 cells treated with TCONS_00068220 siRNA compared with the control group (Fig. 4D).

\section{Discussion}

lncRNAs exhibit diverse functions in the pathogenesis of cancer. The dysregulation of lncRNA is considered to be a critical contributor to thetumorigenesis of various types of cancer, as it mayupregulatetumor cell proliferation, allow the evasion of growth suppressors, enable replicative immortality, induce angiogenesis and increase apoptosis resistance (1). For example, an antisense lncRNA transcribed from the p15 tumor suppressor locus induces alterations to local heterochromatin and DNA methylation status, thereby regulating p15 expression, and may be associated with leukemia oncogenesis (29). TP53-regulated lncRNA (LOC401317) is directly regulated by p53 and has been demonstrated to exhibit antitumor effects in nasopharyngeal carcinoma cells (30). LOC401317 may inhibit cell cycle progression by upregulating p21, and decreasing cyclin D1 and cyclin E1 expression; it may also promote apoptosis through the induction of poly (ADP-ribose) polymerase (PARP) and caspase-3 cleavage (30). Long stress-induced non-coding transcript 5 (LSINCT5) is a $2.6 \mathrm{~kb}$ polyadenylated intergenic nuclear lncRNA that is potentially transcribed by RNA polymerase III. LSINCT5 expression is significantly upregulated in gastrointestinal cancer tissues and cell lines, and is associated with certain clinical pathologies, such as tumor size, tumor invasion depth, lymphatic metastasis and tumor node metastasis stages (31).

Due to advances in microarray production and novel sequencing technologies, the identification and characterization of lncRNAs is now possible. Huang et al (32) investigated the alteration in IncRNA expression induced by hepatitis B virus $\mathrm{X}$ protein $(\mathrm{HBx})$, which wasimplicated as an oncogene in hepatocarcinogenesis, viaepigenetic modification and genetic regulation, based on microarray studies. They identified an lncRNA downregulated by HBx, designated as lncRNA-Dreh, which inhibited hepatocellular carcinoma growth and metastasis by targeting the intermediate filament protein vimentin. Taurine up-regulated gene 1 (TUG1) was initially detected in a genomic screen for genes upregulated in response to taurine 
A

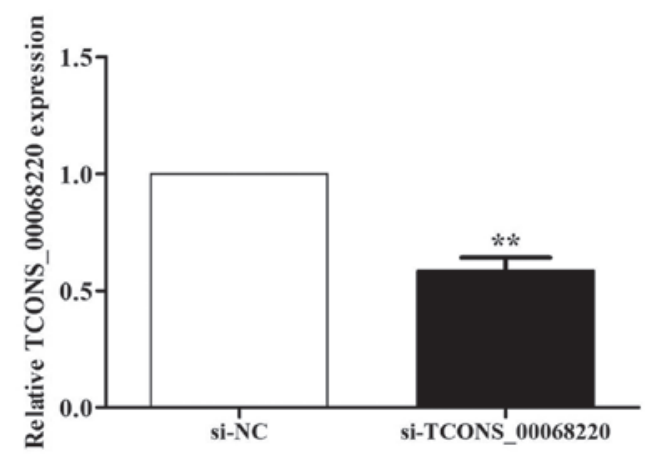

B

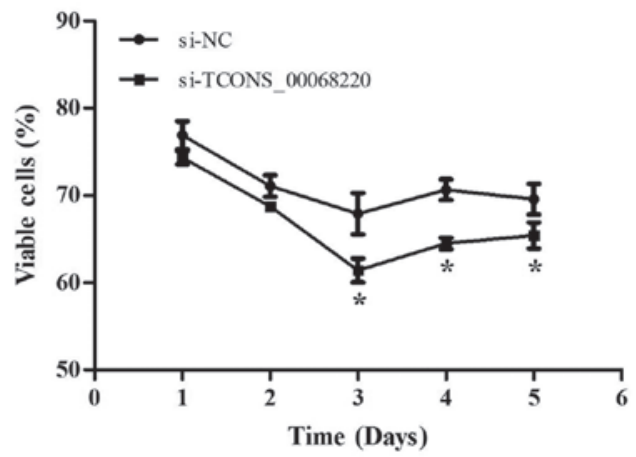

Figure 3. TCONS_00068220 down regulation affects NCI-N87 cell viability. (A) Confirmation of TCONS_00068220 knockdown in NCI-N87 cells following transfection with si-TCONS_00068220, compared with si-NC. (B) The cell viability was determined at each time point witha trypan blue exclusion assay. Data were presented as the mean \pm standard deviation. ${ }^{*} \mathrm{P}<0.05$ and ${ }^{* *} \mathrm{P}<0.01$ compared with si-NC. si-TCONS_00068220, small interfering RNA against TCONS_00068220; si-NC, negative control small interfering RNA.

A

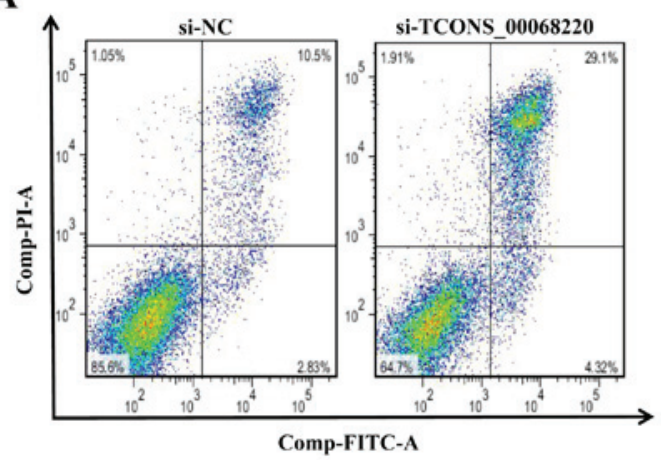

C

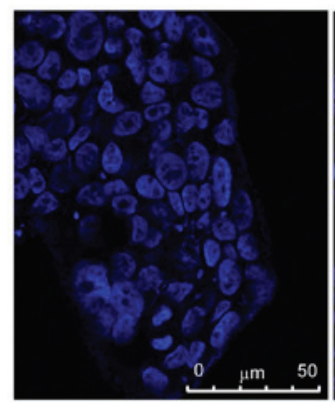

si-NC

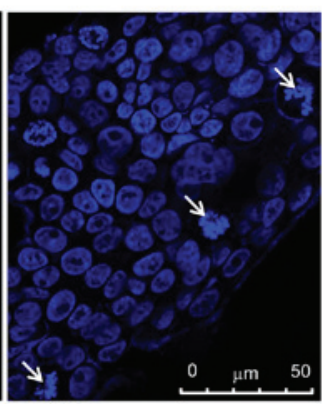

si-TCONS_00068220

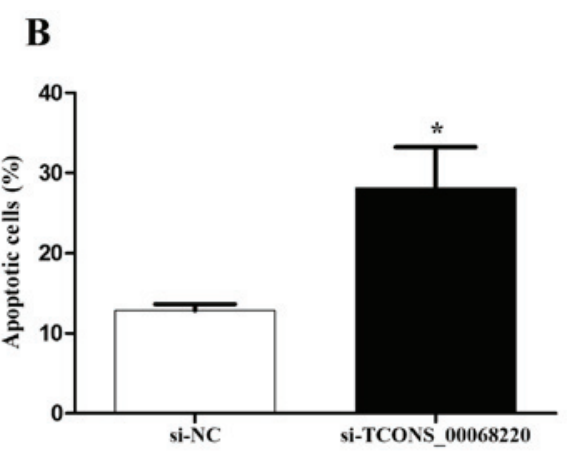

D

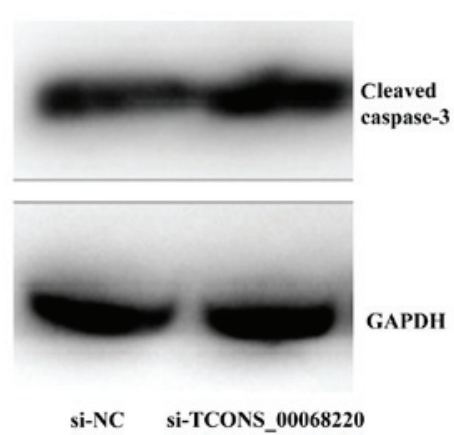

Figure 4. TCONS_00068220 down regulation affects the rate of NCI-N87 cell apoptosis. Following transfection with si-NC or si-TCONS_00068220, changes in the rate of apoptosis were determined by (A) flow cytometry that was (B) quantified, and (C) DAPI staining (white arrows indicate apoptotic cells). (D) The expression of cleaved caspase-3 was detected with western blotting. Flow cytometry data are presented as the mean \pm standard deviation. ${ }^{*}<0.05$ compared with si-NC. si-NC, negative control small interfering RNA; si-TCONS_00068220, small interfering RNA against TCONS_00068220; FITC, fluorescein isothiocyanate.

treatment in developing mouse retinal cells (33). A study demonstrated that the dysregulation of TUG1 is associated with the progression of a variety of tumors. The increased expression of TUG1 predicts a poor prognosis of GC and regulates cell proliferation by epigenetically silencing p57 (34).

In the present study, based on previous data, a DEGs and differentially expressed lncRNAs expression profile of GC tissue samples compared with NATswas screened from RNA-seq data from GEO. A total of 39 lncRNAs were identified as differently expressed. The lncRNA TCONS_00068220 was the focus of the present study; DEGs associated with TCONS_00068220 wereidentified to be enriched in cancer-associated processes. Therefore, TCONS_00068220 was predicted to function in the pathogenesis of GC. It was identified that the expression of TCONS_00068220 was upregulated in GC tissues compared with NATs. In addition, the significantly increased expression of TCONS_00068220 was also identified in GC cell lines. The knockdown of TCONS_00068220 reduced the viability ofNCI-N87 GC cells. To furtherclarify the role of TCONS_00068220 in GC 
cells, a flow cytometry assay was used to detect the apoptosis rate in NCI-N87 cells following transfection with si-NC or si-TCONS_00068220. The results indicated that the down regulation of TCONS_00068220 upregulated the apoptosis of GC cells.

Apoptosisis a genetically regulated 'cellular suicide' mechanism that serves a crucial role in development and homeostasis $(35,36)$. Cancer cells adopt various strategies to override apoptosis, including the upregulation of anti-apoptotic machinery, the down regulation of pro-apoptotic factors or a combination of these strategies (37). A number ofcancer-associated IncRNAs have been identified that affect apoptosis via various pathways $(10,38,39)$. The most prominent pathways for apoptosis are activated by the mitochondria or death receptors from the tumor necrosis factor (TNF) family through various cascade reactions (40). Part of these cascades is the initiator caspases, which activatefurther executorcaspases by cleaving them at aspartate residues. The activation of these executor caspases leads to the activation of further caspases, and ultimately, to cell death by initiating the degradation of DNA and other vital cell components (41). Caspase-3, an executor caspase that serves a central role in the execution of the apoptotic program (42), is primarily responsible for the cleavage of PARP during apoptosis (43). During apoptosis, PARP is cleaved by caspase-3 into 89 - and $24-\mathrm{kDa}$ fragments that contain the active site and the DNA-binding domain of the enzyme, respectively (44-46). This cleavage inactivates PARP by removing its ability to respond to DNA strand breakage (43). Caspase-3 also cleaves Bcl-2 and Bcl-2-extra large, which removes the anti-apoptotic function of these proteins and releases pro-apoptotic C-terminal fragments (47). Caspase-3 also affects the mitochondria; it induces the loss of mitochondrial membrane potential and the release of apoptosis inducing factor (28). In the present study, following the down regulation of TCONS_00068220, the level of cleaved caspase-3 was markedly increased, which implies TCONS_00068220 may serve a role in preventing the apoptosis of cancer cells.

In summary, a DEGs and differentially expressed lncRNAs expression profile of GC samples relative to normal tissue samples was screened with bioinformatics methods. TCONS_00068220 was identified as a novel lncRNA associated with apoptosis inhibition in GC cells. These data suggest that TCONS_00068220 may serve a key functional role in GC occurrence and progression. To the best of our knowledge, this is the first study to examine the biological functions of TCONS_00068220. Therefore, additional detailed study is required.

\section{Acknowledgements}

The authors wish to thank Ms. Qimeng Yuan (First Affiliated Hospital of Harbin Medical University, Harbin, China) and Dr Fengqi Jiang (People's Hospital of Heilongjiang Province, Harbin, China) for gifting the tissue samples.

\section{References}

1. Fang XY, Pan HF, Leng RX and Ye DQ: Long noncoding RNAs: Novel insights into gastric cancer. Cancer Lett 356: 357-366, 2015.
2. Jemal A, Bray F, Center MM, Ferlay J, Ward E and Forman D: Global cancer statistics. CA Cancer J Clin 61: 69-90, 2011.

3. Sun M, Xia R, Jin F, Xu T, Liu Z, De W and Liu X: Downregulated long noncoding RNA MEG3 is associated with poor prognosis and promotes cell proliferation in gastric cancer. Tumor Biol 35: 1065-1073, 2014.

4. Chen X, Sun J, Song Y, Gao P, Zhao J, Huang X, Liu B, Xu H and Wang Z: The novel long noncoding RNA AC138128.1 may be a predictive biomarker in gastric cancer. Med Oncol 31: 262, 2014.

5. Guo X, Xia J and Deng K: Long non-coding RNAs: Emerging players in gastric cancer. Tumor Biol 35: 10591-10600, 2014.

6. Zhao JH, Sun JX, Song YX, Chen XW, Yang YC, Ma B, Wang J, Gao P and Wang ZN: A novel long noncoding RNA-LOWEG is low expressed in gastric cancer and acts as a tumor suppressor by inhibiting cell invasion. J Cancer Res Clin Oncol 142: 601-609, 2016.

7. Ding J, Lu B, Wang J, Shi Y, Lian Y, Zhu Y, Wang J, Fan Y, Wang Z, De W and Wang K: Long non-coding RNA Loc554202 induces apoptosis in colorectal cancer cells via the caspase cleavage cascades. J Exp Clin Cancer Res 34: 100, 2015.

8. Luo G, Wang M, Wu X, Tao D, Xiao X, Wang L, Min F, Zeng F and Jiang G: Long non-coding RNA MEG3 inhibits cell proliferation and induces apoptosis in prostate cancer. Cell Physiol Biochem 37: 2209-2220, 2015.

9. Ponting CP, Oliver PL and Reik W: Evolution and functions of long noncoding RNAs. Cell 136: 629-641, 2009.

10. Zhu H, Li X, Song Y, Zhang P, Xiao Y and Xing Y: Long non-coding RNA ANRIL is up-regulated in bladder cancer and regulates bladder cancer cell proliferation and apoptosis through the intrinsic pathway. Biochem Biophys Res Commun 467: 223-228, 2015.

11. Zang W, Wang T, Wang Y, Chen X, Du Y, Sun Q, Li M, Dong Z and Zhao G: Knockdown of long non-coding RNA TP73-AS1 inhibits cell proliferation and induces apoptosis in esophageal squamous cell carcinoma. Oncotarget 7: 19960-19974, 2016.

12. Zhu S, Mao J, Shao Y, Chen F, Zhu X, Xu D, Zhang X and Guo J: Reduced expression of the long non-coding RNA AI364715 in gastric cancer and its clinical significance. Tumor Biol 36: 8041-8045, 2015.

13. Yang F, Bi J, Xue X, Zheng L, Zhi K, Hua J and Fang G: Up-regulated long non-coding RNA H19 contributes to proliferation of gastric cancer cells. FEBS J 279: 3159-3165, 2012.

14. Li H, Yu B, Li J, Su L, Yan M, Zhu Z and Liu B: Overexpression of lncRNA H19 enhances carcinogenesis and metastasis of gastric cancer. Oncotarget 5: 2318-2329, 2014.

15. Kim KS and Lee YI: Biallelic expression of the H19 and IGF2 genes in hepatocellular carcinoma. Cancer Lett 119: 143-148, 1997.

16. Luo M, Li Z, Wang W, Zeng Y, Liu Z and Qiu J: Upregulated H19 contributes to bladder cancer cell proliferation by regulating ID2 expression. FEBS J 280: 1709-1716, 2013.

17. Berteaux N, Lottin S, Monté D, Pinte S, Quatannens B, Coll J, Hondermarck H, Curgy JJ, Dugimont T and Adriaenssens E: H19 mRNA-like noncoding RNA promotes breast cancer cell proliferation through positive control by E2F1. J Biol Chem 280: 29625-29636, 2005.

18. Hibi K, Nakamura H, Hirai A, Fujikake Y, Kasai Y, Akiyama S, Ito A and Takagi H: Loss of H19 imprinting in esophageal cancer. Cancer Res 56: 480-482, 1996.

19. Wang J, Song YX and Wang ZN: Non-coding RNAs in gastric cancer. Gene 560: 1-8, 2015.

20. Niu H, Hu Z, Liu H, Hu G, Yang B, Wu S and Li F: Long non-coding RNA AK027294 involves in the process of proliferation, migration, and apoptosis of colorectal cancer cells. Tumor Biol 37: 10097-10105, 2016.

21. Li PF, Chen SC, Xia T, Jiang XM, Shao YF, Xiao BX and Guo JM: Non-coding RNAs and gastric cancer. World J Gastroenterol 20: 5411-5419, 2014.

22. Song H, Sun W, Ye G, Ding X, Liu Z, Zhang S, Xia T, Xiao B, $\mathrm{Xi} \mathrm{Y}$ and Guo J: Long non-coding RNA expression profile in human gastric cancer and its clinical significances. J Transl Med 11: 225, 2013

23. Sun W, Wu Y, Yu X, Liu Y, Song H, Xia T, Xiao B and Guo J: Decreased expression of long noncoding RNA AC096655.1-002 in gastric cancer and its clinical significance. Tumor Biol 34: 2697-2701, 2013

24. Park SM, Park SJ, Kim HJ, Kwon OH, Kang TW, Sohn HA, Kim SK, Moo Noh S, Song KS, Jang SJ, et al: A known expressed sequence tag, BM742401, is a potent lincRNA inhibiting cancer metastasis. Exp Mol Med 45: e31, 2013. 
25. Zhao Z, Song Y, Piao D, Liu T and Zhao L: Identification of genes and long non-coding RNAs associated with the pathogenesis of gastric cancer. Oncol Rep 34: 1301-1310, 2015.

26. Livak KJ and Schmittgen TD: Analysis of relative gene expression data using real-time quantitative PCR and the 2(-Delta Delta C(T)) method. Methods 25: 402-408, 2001.

27. Liao X, Tang S, Thrasher B, Griebling TL and Li B: Small-interfering RNA-induced androgen receptor silencing leads to apoptotic cell death in prostate cancer. Mol Cancer Ther 4: 505-515, 2005.

28. Lakhani SA, Masud A, Kuida K, Porter GA Jr, Booth CJ, Mehal WZ, Inayat I and Flavell RA: Caspase 3 and 7: Key mediators of mitochondrial events of apoptosis. Science 311: 847-851, 2006.

29. Mercer TR, Dinger ME and Mattick JS: Long non-coding RNAs: Insights into functions. Nat Rev Genet 10: 155-159, 2009.

30. Gong Z, Zhang S, Zeng Z, Wu H, Yang Q, Xiong F, Shi L, Yang J, Zhang W, Zhou Y, et al: LOC401317, a p53-regulated long non-coding RNA, inhibits cell proliferation and induces apoptosis in the nasopharyngeal carcinoma cell line HNE2. PLoS One 9: e110674, 2014.

31. Xu MD, Qi P, Weng WW, Shen XH, Ni SJ, Dong L, Huang D, Tan C, Sheng WQ, Zhou XY and Du X: Long non-coding RAN LSINCT5 predicts negative prognosis and exhibits oncogenic activity in Gastric cancer. Medicine (Baltimore) 93: e303, 2014.

32. Huang JF, Guo YJ, Zhao CX, Yuan SX, Wang Y, Tang GN, Zhou WP and Sun SH: Hepatitis B virus X protein $(\mathrm{HBx})$-related long noncoding RNA (lncRNA) down-regulated expression by HBx (Dreh) inhibits hepatocellular carcinoma metastasis by targeting the intermediate filament protein vimentin. Hepatology 57: 1882-1892, 2013

33. Young TL, Matsuda T and Cepko CL: The noncoding RNA taurine upregulated gene 1 is reguired for differentiation of the murine retina. Curr Biol 15: 501-512, 2005.

34. Zhang E, He X, Yin D, Han L, Qiu M, Xu T, Xia R, Xu L, Yin R and De W: Increased expression of long noncoding RNA TUG1 predicts a poor prognosis of gastric cancer and regulates cell proliferation by epigenetically silencing of p57. Cell Death Dis 7: e2109, 2016.

35. Cryns V and Yuan JY: Proteases to die for. Gene \& Development 12: 1551-1570, 1998.

36. Earnshaw WC, Martins LM and Kaufmann SH: Mammalian caspases: Structure, activation, substrates, and functions during apoptosis. Annu Rev Biochem 68: 383-424, 1999.
37. Fernald $\mathrm{K}$ and Kurokawa M: Evading apoptosis in cancer. Trends cell Biol 23: 620-633, 2013.

38. Wang M, Huang T, Luo G, Huang C, Xiao XY, Wang L, Jiang GS and Zeng FQ: Long non-coding RNA MEG3 induces renal cell carcinoma cells apoptosis by activating the mitochondrial pathway. J Huazhong Univ Sci Technolog Med Sci 35: 541-545, 2015.

39. Zhu YP, Bian XJ, Ye DW, Yao XD, Zhang SL, Dai B, Zhang HL and Shen YJ: Long noncoding RNA expression signatures of bladder cancer revealed by microarray. Oncol Lett 7: 1197-1202, 2014.

40. Daniel PT: Dissecting the pathways to death. Leukemia 14: 2035-2044, 2000.

41. Juraver-Geslin HA and Durand BC: Early development of the neural plate: New roles for apoptosis and for one of its main effectors caspase-3. Genesis 53: 203-224, 2015.

42. Friedrich K, Wieder T, Von Haefen C, Radetzki S, Jänicke R, Schulze-Osthoff K, Dörken B and Daniel PT: Overexpression of caspase-3 restores sensitivity for drug-induced apoptosis in breast cancer cell lines with acquired drug resistance. Oncogene 20: 2749-2760, 2001.

43. Boulares AH, Yakovlev AG, Ivanova V, Stoica BA, Wang G, Iyer S and Smulson M: Role of poly(ADP-ribose) polymerase (PARP) cleavage in apoptosis. Caspase 3-resistant PARP mutant increases rates of apoptosis in transfected cells. J Biol Chem 274: 22932-22940, 1999.

44. Tewari M, QuanLT,O'RourkeK,Desnoyers S,Zeng Z, BeidlerDR, Poirier GG, Salvesen GS and Dixit VM: Yama/CPP32beta, a mammalian homolog of CED-3, is a CrmA-inhibitable protease that cleaves the death substrate poly(ADP-Ribose) polymerase. Cell 81: 801-809, 1995.

45. Liu J, Wu Y, Wang B, Yuan X and Fang B: High levels of glucose induced the caspase-3/PARP signaling pathway, leading to apoptosis in human periodontal ligament fibroblasts. Cell Biochem Biophys 66: 229-237, 2013.

46. Krishnakumar R and Kraus WL: The PARP side of the nucleus: Molecular actions, physiological outcomes, and clinical targets. Mol Cell 39: 8-24, 2010.

47. Wolf BB and Green DR: Suicidal tendencies: Apoptotic cell death by caspase family proteinases. J Biol Chem 274: 20049-20052, 1999. 